\title{
The flavonoid-rich Quzhou Fructus Aurantii extract modulates gut microbiota and prevents obesity in high-fat diet-fed mice
}

Yong-feng Bai', Si-wei Wang ${ }^{2,3}$, Xiao-xiao Wang ${ }^{4}$, Yuan-yuan Weng ${ }^{1}$, Xue-yu Fan ${ }^{1}$, Hao Sheng ${ }^{5}$, Xin-tian Zhu ${ }^{6}$, Li-jun Lou ${ }^{3}$ and Feng Zhang (10)

\begin{abstract}
Background: Flavonoids are reported to modulate the composition of gut microbiota, which play an important role in preventing obesity and associated metabolic diseases. In this study, we investigated the effect of Total Flavonoids of Quzhou Fructus Aurantii Extract (TFQ) on gut microbial community in mice fed with a high-fat diet (HFD).
\end{abstract}

Methods: C57BL/6J mice were fed with either a chow diet or HFD with or without oral gavage of TFQ (300 mg/kg/ day) for 12 weeks.

Results: Our data indicate TFQ significantly reduced obesity, inflammatio,n and liver steatosis. TFQ elevates the expression of tight junction proteins and reduces metabolic endotoxemia. In addition, TFQ treatment reverses HFDinduced gut dysbiosis, as indicated by the reduction of Firmicutes to Bacteroidetes ratio, the increase of genera Akkermansia and Alistipes, and the decrease of genera Dubosiella, Faecalibaculum, and Lactobacillus.

Conclusion: These findings support a prebiotic role of TFQ as a dietary supplement for the intervention of gut dysbiosis and obesity-related metabolic disorders.

\section{Introduction}

Obesity is a growing public health concern which strongly influences the quality of human life ${ }^{1}$. Growing evidence indicates that chronic inflammation caused by obesity is an independent risk factor for chronic diseases such as insulin resistance, nonalcoholic fatty liver disease and inflammatory bowel disease, and it significantly increases the morbidity and mortality of these diseases ${ }^{2-4}$.

In recent years, with the advances in human microbiota research, accumulating evidence shows that gut microbiota dysbiosis is tightly associated with obesity ${ }^{5,6}$. Gut

\footnotetext{
Correspondence: Li-jun Lou (Iljqz@aliyun.com) or Feng Zhang

(felix.f.zhang@outlook.com)

'Department of Clinical Laboratory, Quzhou People's Hospital, 324000 Quzhou, China

Department of Core Facility, Quzhou People's Hospital, 324000 Quzhou, China Full list of author information is available at the end of the article.

These authors contributed equally: Yong-feng Bai, Si-wei Wang
}

microbiota is involved in regulating various physiological metabolic processes, which are vital to the digestion, nutrition, metabolism, and immunity of our bodies ${ }^{7}$. The dysbiosis of gut microbiota, which is mostly manifested as a decrease in bacterial richness and diversity, may induce low-grade inflammation, leading to metabolic diseases such as obesity ${ }^{8,9}$. Studies have shown that high-fat dietinduced obesity disrupts mucosal barrier integrity and leaks bacterial lipopolysaccharide (LPS) from the intestinal lumen into bloodstream, leading to metabolic endotoxemia and body weight gain ${ }^{7,10}$. Therefore, modulation of gut microbiota may represent a novel therapeutic approach for obesity and metabolic syndrome treatment.

Flavonoids are important dietary supplements with antiobesity and anti-diabetic properties ${ }^{11}$. The clinical and experimental studies have provided compelling evidence that flavonoids intake aids in preventing obesity and type 
2 diabetes ${ }^{12,13}$. Flavonoids can be transformed by gut microbes into metabolites with increased or decreased biological activities ${ }^{14}$. Meanwhile, flavonoids such as hesperidin ${ }^{15}$, quercetin ${ }^{16}$, and Cyclocarya paliurus flavonoid $^{17}$, have been reported to modulate the composition of gut microbiota by increasing probiotics and reducing pathogens, which play an important role in preventing obesity and associated metabolic diseases. The complex interaction between flavonoids and gut microbiota is essential for the pharmacological activities of these natural products ${ }^{18}$.

Quzhou Fructus Aurantii is a dried unripe fruit of Rutaceae Citrus changshan-huyou, which is recorded in the "Zhejiang Traditional Chinese Medicine Processing Norms (2015)"19. Our previous study has shown that the main components of Quzhou Fructus Aurantii are naringin, narirutin, hesperidin and neohesperidin, which constitute an important sort of flavonoids ${ }^{20}$. In the practice of traditional Chinese medicine, Quzhou Fructus Aurantii is often used in the treatment of gastrointestinal diseases. However, the effect of Quzhou Fructus Aurantii on gut microbiota has not been reported. In this study, we investigated the influence of the Quzhou Fructus Aurantii extract, $60-70 \%$ of which consisting of flavonoids, on gut microbiota in a high-fat diet-fed mice model. Our results, for the first time, demonstrate that the flavonoid-rich Quzhou Fructus Aurantii extract ameliorates fatty liver, insulin resistance and intestinal inflammation through the modification of gut microbiota at least in part.

\section{Materials and methods}

\section{The total flavonoids of Quzhou Fructus Aurantii Extract} (TFQ)

Quzhou Fructus Aurantii was purchased from Quzhou Nankong Chinese Medicine Co., Ltd. The dried Quzhou Fructus Aurantii was crushed and extracted three times with ethanol/water $(80: 20, \mathrm{v} / \mathrm{v})$ at $60^{\circ} \mathrm{C}$ for $2 \mathrm{~h}$ each time. Then the extract was filtered and freeze-dried. The flavonoid composition in Quzhou Fructus Aurantii extract was determined by high performance liquid chromatography (HPLC). The characterizations of its flavonoids are shown in Table 1.

\section{Animal experiments}

Animal experiments were approved and performed in accordance with the guidelines of Committee on the Ethics of Animal Experiments of Zhejiang University of Traditional Chinese Medicine, China. Thirty-six 8-weekold C57BL/6J male mice (Shanghai Laboratory Animal Center) were housed under standard pathogen-free conditions with controlled light conditions (a light-dark cycle $12 \mathrm{~h}$ ) and food and water ad libitum. After 2 weeks of acclimation, 36 mice were randomly distributed into three groups of 12 mice (4 mice per cage): (a) Chow group, which fed a chow diet; (b) high- fat diet (HFD) group, which fed a HFD with $60 \mathrm{kcal} \%$ fat (Research diet D12492, Research Diet, NJ); (c) TFQ group, which fed HFD and daily dose of $300 \mathrm{mg} / \mathrm{kg}$ TFQ by gavage for 12 weeks. Body weight gain and food intake were assessed once a week. At the end of the experiment, the samples of colonic contents for microbiological examination were collected.

\section{Glucose homeostasis}

For insulin tolerance tests (ITT), at week 7, mice were $6 \mathrm{~h}$ fasted and injected i.p. with insulin $(0.75 \mathrm{U} / \mathrm{kg}$ body weight). An automatic glucometer (OneTouch Ultra, Lifescan, Milpitas, CA) measured tail vein blood glucose at $0,30,60,90$, and $120 \mathrm{~min}$ after injection. Oral glucose tolerance tests (OGTT) were performed after $12 \mathrm{~h}$ fasts at the end of week 8 . The glucose $(2 \mathrm{~g} / \mathrm{kg}$ body weight) was delivered by gastric gavage. The glucose levels were monitored at $0,15,30,60,90$, and $120 \mathrm{~min}$ as before mentioned.

\section{Biochemical testing}

Serum alanine transaminase (ALT), aspartate transaminase (AST), total cholesterol (TC), triglyceride (TG), high density lipoprotein cholesterol (HDL-C), low density lipoprotein cholesterol (LDL-C) and non-esterified fatty acids (NEFA) were measured according to the manufacturer's instruction (DiaSys Diagnostic Systems, Shanghai, China). Serum insulin and lipopolysaccharides (LPS) concentration, was tested by enzyme-linked immunosorbent assay (ELISA) kits according to the manufacturer's instructions (MEIMIAN, China). The homeostasis model

Table 1 Chemical characterization of Quzhou Fructus Aurantii extract

\begin{tabular}{lcc}
\hline Flavonoid composition & Content (\%) & Daily intake (mg/kg body weight) \\
\hline Narirutin & $4.57 \pm 0.06$ & $13.71 \pm 0.18$ \\
Naringin & $41.00 \pm 0.35$ & $122.91 \pm 1.19$ \\
Hesperidin & $2.63 \pm 0.08$ & $7.89 \pm 0.24$ \\
Neohesperidin & $20.61 \pm 0.12$ & $61.83 \pm 0.36$ \\
\hline
\end{tabular}


assessment of insulin resistance (HOMA-IR) index was calculated according to the formula: fasting insulin $(\mathrm{mU} / \mathrm{ml}) \times$ fasting glucose $(\mathrm{mmol} / \mathrm{L}) / 22.5$. Liver triglyceride (TG), as well as cholesterol (TC) content was assessed using enzymatic reactions with commercial kits (Dongou Diagnostics Co., LTD, Zhejiang, China).

\section{Pathological staining}

Liver and epididymal white adipose tissues were carefully collected for hematoxylin-eosin (H\&E) staining. Non-alcoholic fatty liver disease (NAFLD) activity score is the unweighted sum of steatosis, lobular inflammation and hepatocellular ballooning score according to the Brunt system ${ }^{21}$.

\section{Quantitative real-time PCR (qPCR)}

Total RNA was isolated using TRIzol reagent (Tiangen Biotech, China) according to the manufacturer's instructions. RNA concentration was evaluated by absorbance at 260 and $280 \mathrm{~nm}$ using Nano-100 microscope spectrophotometer (Allsheng Instruments, China). Complementary DNA (cDNA) synthesis was performed using reverse transcriptase kits (Thermo Fisher Scientific, Waltham, MA) following the manufacturer's instructions. Realtime PCR was performed using SYBR Green (Sangon Biotech, China) on LightCycler 480 instrument (Roche, Basel, Switzerland). The relative gene quantities were calculated by the $2^{-\Delta \Delta C t}$ method in comparison with the expression levels of GAPDH. The primers are listed in Table 2.

\section{Western blotting}

Western blotting was performed as described previously $^{22,23}$. Protein from each colon sample was extracted with lysis buffer (50 mM Tris-HCl, pH 7.4, 2 mM EDTA, $150 \mathrm{mM} \mathrm{NaCl}, 0.1 \%$ sodium dodecyl sulfate, and 1\% NP40), supplemented with protease inhibitor cocktail and phosphatase inhibitor cocktail $(1 \mathrm{mM}$ sodium orthovanadate, $5 \mathrm{mM}$ sodium fluoride, $3 \mathrm{mM} \beta$-glycerophosphate, and $4 \mathrm{mM}$ sodium tartrate) at $4{ }^{\circ} \mathrm{C}$. Then the extracts were centrifuged at $4{ }^{\circ} \mathrm{C}$ for $10 \mathrm{~min}$ at $16,000 \times g$ and separated supernatant for western blotting analyses. The equal amounts of proteins boiled for $5 \mathrm{~min}$, then separated by $10-15 \%$ SDS-PAGE and transferred to PVDF membranes. The membranes were blocked in $1 \%$ casein for detection of proteins, incubated overnight at $4{ }^{\circ} \mathrm{C}$ in primary antibodies and visualized with secondary HRP-conjugated antibodies. Immune complexes were detected by the Tanon 4200SF system from Tanon Biotechnology (Shanghai, China). Band intensity was quantified using ImageJ software (U.S. National Institutes of Health, Bethesda, MD). The primary antibodies used in this study were anti-phospho-NF- $\kappa B$ p65 (1:1000, \#3033, Cell Signaling Technology), anti-phospho-IKK $\alpha / \beta$ (1:1000, \#2697, Cell Signaling Technology), anti-TNF- $\alpha$ (1:500, ab183218, Abcam), anti-COX-2 (1:500, ab179800, Abcam), and anti$\beta$-actin (1:3000, A1978, Millipore Sigma).

\section{Gut microbiota analysis}

The genomic DNA of colonic content was extracted with modified CTAB method. V3, V4 hypervariable regions of microbial $16 \mathrm{~S}$ rRNA genes were amplified using the MetaVxTM Library Preparation kit (GENEWIZ, Inc., South Plainfield, NJ.USA). Sequencing was accomplished on the Illumina HiSeq platform by GENEWIZ, Inc. (Suzhou, China) using a method described previously ${ }^{24}$. Sequences were clustered into operational taxonomic units (OTUs) using a 97\% identity cutoff. The Ribosomal Database Project (RDP) classifier uses a Bayesian approach to assign 16S rRNA sequences into different taxonomic levels. MicrobiomeAnalyst ${ }^{25}$, a web-based tool, was used to calculate alpha diversity analysis, principal coordinate analysis (Bray-Curtis distances) and comparative analysis of microbiome data. Biomarker discovery using Linear Discriminant Analysis Effect Size (LEfSe) was performed online (http://huttenhower.sph.harvard.edu/ galaxy).

\section{Statistical analysis}

Data are graphed as mean and error bars show standard deviation (S.D.) unless otherwise stated. All data were analyzed using one way analysis of variance (ANOVA) by SPSS software (version 20.0) or MicrobiomeAnalyst program. Differences were considered to be statistically significant at $P<0.05$ and highly significant at $P<0.01$.

\section{Results}

TFQ ameliorates obesity and fatty liver and improves insulin resistance in HFD-fed mice

As depicted in Fig. 1a and S1A, compared to chow-fed mice, the mice gained significantly more body weight after

Table 2 The primers used in this study for real time PCR

\begin{tabular}{lll}
\hline Description & Sense primer $\left(\mathbf{5}^{\prime} \rightarrow \mathbf{3}^{\prime}\right)$ & Antisense primer $\left(\mathbf{5}^{\prime} \boldsymbol{\rightarrow} \mathbf{3}^{\prime}\right)$ \\
\hline Cldn3 & CAGGGGCAGTCTCTGTGCGAG & GCCGCTGGACCTGGGAATCAAC \\
Ocln & ATGTCCGGCCGATGCTCTC & TTGGCTGCTCTTGGGTCTGTAT \\
GAPDH & TGAGGCCGGTGCTGAGTATGT & CAGTCTTCTGGGTGGCAGTGAT \\
\hline
\end{tabular}



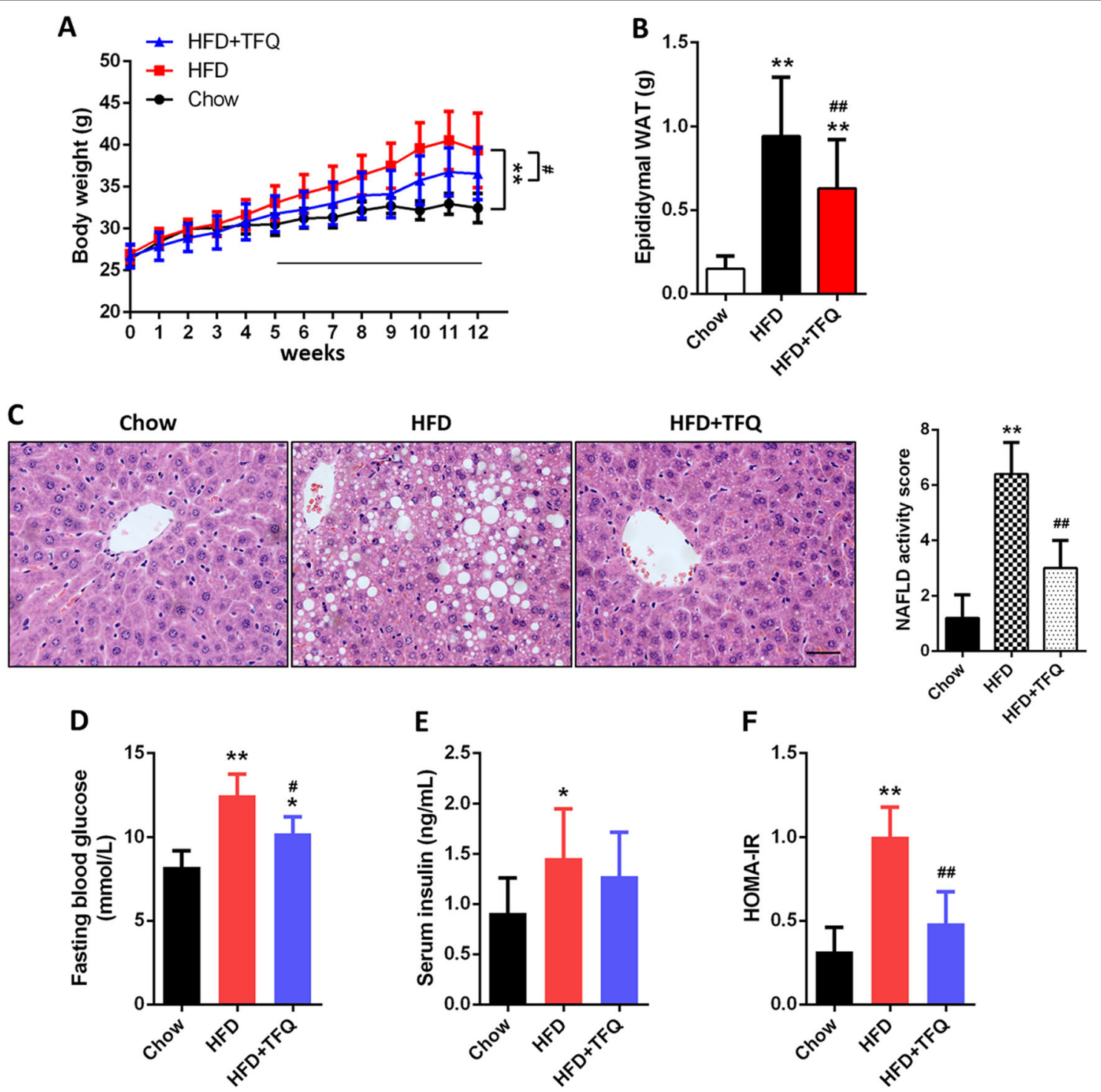

Fig. 1 The effect of TFQ on obesity, fatty liver and insulin resistance in HFD-fed mice. C57BL/6J mice were fed either a chow or a high-fat diet (HFD) for 12 weeks. Mice were treated with daily oral doses of TFQ $(300 \mathrm{mg} / \mathrm{kg})$. Water was gavaged as control. a Body weight curve $(n=12)$. b Epididymal white adipose tissue (WAT) weight $(n=12)$. c Left, the representative images of H\&E staining in liver from each group. Scale bar, $300 \mu m$. Right, NAFLD activity scores in each group $(n=5)$. $\mathbf{d}$ Fasting blood glucose level in last week $(n=12)$. e Serum insulin level $(n=12)$. $\mathbf{f}$ Homeostasis model assessment of insulin resistance $(H O M A-I R)$ index $(n=12)$. Data were expressed as the mean \pm SD. ${ }^{*} p<0.05$, ${ }^{* *} p<0.01$, vs. chow group; ${ }^{*} p<$ $0.05,{ }^{\# \#} p<0.01$, vs. HFD group

5-week HFD feeding. HFD feeding also resulted in marked increase of the epididymal white adipose tissue (WAT) mass (Fig. 1b and S1B). TFQ gavage at a dose of $300 \mathrm{mg} / \mathrm{kg}$ significantly reduced HFD-induced weight gain of the whole body and the epididymal WAT (Fig. 1a, b). The H\&E staining of epididymal WAT revealed that the administration of TFQ prominently attenuated HFDinduced adipocyte hypertrophy and hyperplasia. (Fig. S1B).
After 12-week HFD feeding, mice developed obvious hyperlipidemia and fatty liver, reflected by elevated TC and TG levels in serum and liver tissue. As shown in Fig. S2A and S2B, the serum TC and TG levels were evidently increased in HFD-fed mice compared to normal chow counterparts. These indicators were significantly decreased after treatment with TFQ. TFQ also reduced HFD-induced serum NEFA and LDL-C, but had no effect on HDL-C level in these mice (Fig. S2C-E). 
Moreover, administration of HFD-fed mice with TFQ decreased hepatic TG accumulation and liver weight, concomitantly reducing serum ALT and AST, while had no effect on hepatic TC (Fig. S3). The results of H\&E staining showed that the HFD induced obvious hepatic lobular disorders, hepatic steatosis and hepatocellular ballooning with inflammatory cell infiltrations in mice. TFQ administration ameliorated all these liver histopathological changes and decreased the NAFLD activity scores (Fig. 1c).

Insulin resistance is one of the commonest pathological features in HFD fed mice ${ }^{26}$. As expected, HFD feeding markedly elevated fasting blood glucose (FBG) (Fig. 1d) and insulin levels (Fig. 1e). Although TFQ treatment did not significantly lower insulinemia in HFD-fed mice, it obviously decreased FBG and HOMA-IR levels (Fig. 1d and f), suggesting that TFQ ameliorated insulin sensitivity in HFD-fed mice. We next conducted oral glucose-tolerance tests (OGTT) and insulin-tolerance tests (ITT) to further assess the effect of TFQ on glucose homeostasis and insulin sensitivity. The administration of TFQ lessened values of OGTT in HFD-fed mice (Fig. S4A). In addition, TFQ treated mice displayed a downward trend $(P=0.0632)$ in AUC-ITT compared to HFD-fed model group (Fig. S4B).

\section{TFQ suppresses serum LPS level and intestinal} inflammation in HFD-fed mice

It has been reported that HFD could change intestinal permeability and cause low-grade intestinal inflammation through raising circulating LPS level ${ }^{27}$. As shown in Fig. 2a, 12 weeks of HFD feeding significantly raised serum LPS level, which was reduced by TFQ administration. The expression of Cldn3 and Ocln in colon tissues, which are major components of tight junction and play key roles in regulating gut permeability ${ }^{28}$, was significantly lower in HFD fed mice and remarkably recovered after TFQ treatment (Fig. 2b). To further determine the effect of TFQ on intestinal inflammation induced by HFD, we also examined the expression of intestinal inflammatory cytokines in mice. The results showed that TFQ administration markedly inhibited the phosphorylation of NF$\kappa B$ p65 and IKK $\alpha / \beta$, as well as the amount of TNF- $\alpha$ and COX-2 $(P<0.05)$ (Fig. 2c, d).

\section{TFQ modulates the overall composition of intestinal bacterial community}

The intestinal flora is crucial in the pathogenesis of obesity and related metabolic diseases. To investigate whether TFQ regulated gut microbiota of HFD-fed mice, we performed $16 \mathrm{~S}$ rRNA sequencing to analysis of the bacterial community structure in specimen of intestinal contents. The Venn diagram showed the numbers of OTUs detected in each group. There are 202 OTUs shared by three groups. Meanwhile, each group had unique OTUs
(Fig. 3a). Treatment with TFQ significantly increased the alpha diversity of the gut microbiota as indicated by the Chao1 index and the Shannon index (Fig. 3b, c). Principal coordinate analysis (PCoA) was used to determine clustering patterns among three groups. The gut microbiota of the TFQ group was closer to the control group in PCoA plot (Fig. 3d). These results showed that TFQ treatment improved the structure of the intestinal flora.

\section{TFQ manipulates the specific phylotypes of the gut microbiota}

The histograms illustrating the gut microbiota community structure revealed the microbial species and their relative abundance at the phylum level (Fig. 4a). The proportion of Verrucomicrobia and Bacteroidetes was markedly decreased, whereas the relative abundance of Actinobacteria was significantly increased in the HFD-fed mice compared to their chow-fed counterparts. In contrast, TFQ treatment corrected relative abundance of these bacterial groups (Fig. 4b). Firmicutes and Bacteroidetes are the two most abundant bacterial phyla in the intestinal tracts of experimental mice. The gut microbiota of HFD-fed mice was characterized by a dramatic increase of the Firmicutes to Bacteroidetes ratio compared with that of the chow-diet group, and this trend was also reversed by the TFQ treatment (Fig. 4c).

Figure 5 displays the abundance changes of the top 10 genera among the three different groups. At the genus level, HFD feeding reduced Akkermansia and Alistipes and enlarged Dubosiella, Faecallbaculum, and Lactobacillus. Notably, TFQ could partly reverse these changes. These obvious alterations of individual genera after TFQ treatment not only confirmed the modulatory effect of TFQ on the gut microbiota but also implied that these genera might be key bacteria to the therapeutic effects of TFQ.

\section{TFQ changes taxonomic biomarkers in each group}

In addition, to identify the characteristic bacteria which were specific for each group, linear discriminant analysis (LDA) effect size (LEfSe) algorithm approach was applied. The chow group showed the most unique microbiota by a high abundance of genera Ambiguous-taxa (class within Bacteroidia) and Akkermansia (class within Verrucomicrobiae). The genera of Faecallbaculum and Dubosiella (class within Erysipelotrichia) and family Atopotiaceae (class within Coriobacteriia) were the dominant phylotypes that contributed to the differences between the intestinal microbiota of chow and HFD-fed mice. The abundance of genus Blautia (class within Clostridia) was higher in the TFQ group (Fig. 6).

\section{Discussion}

It is believed that flavonoids and their metabolites could protect against obesity-related diseases ${ }^{12,18}$. Given that 

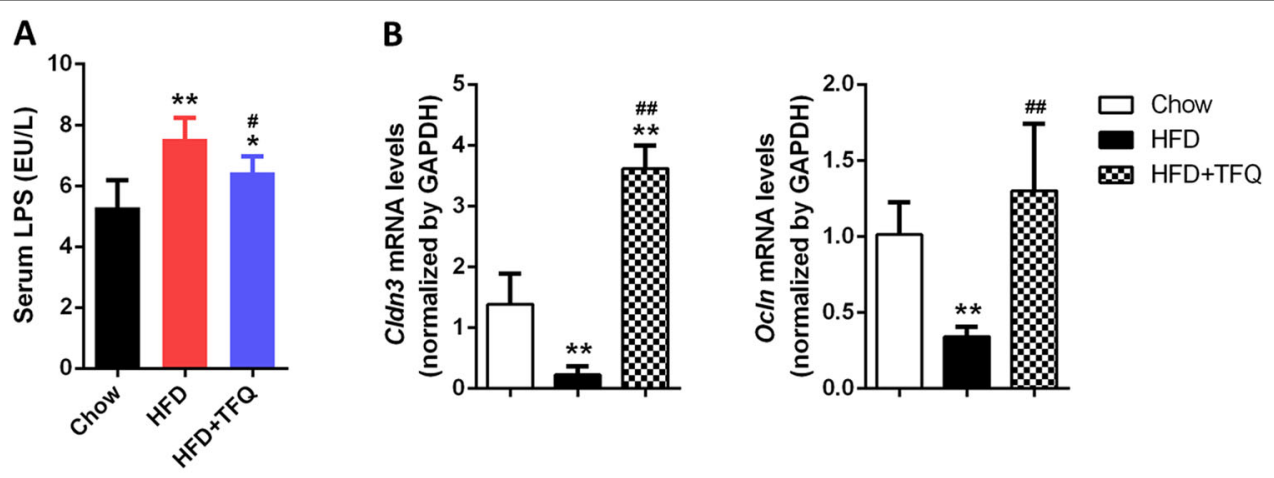

C
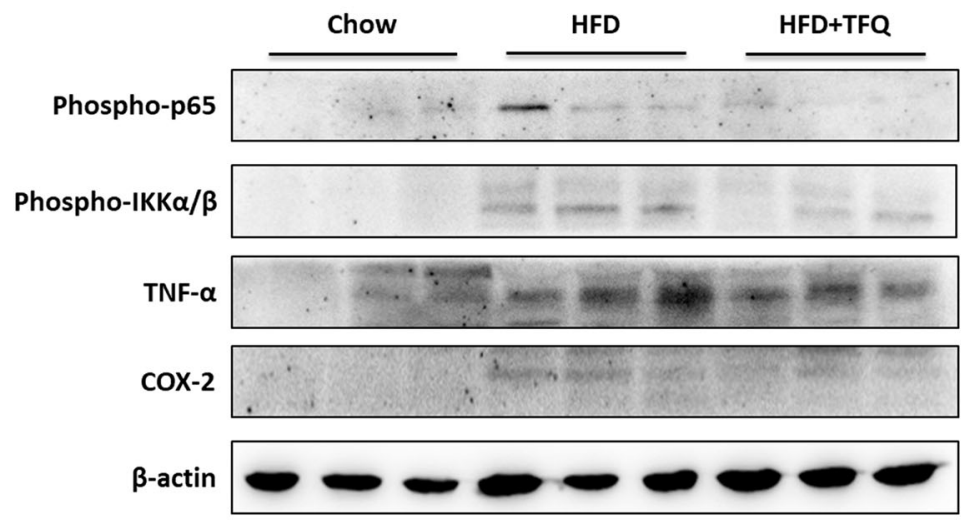

D

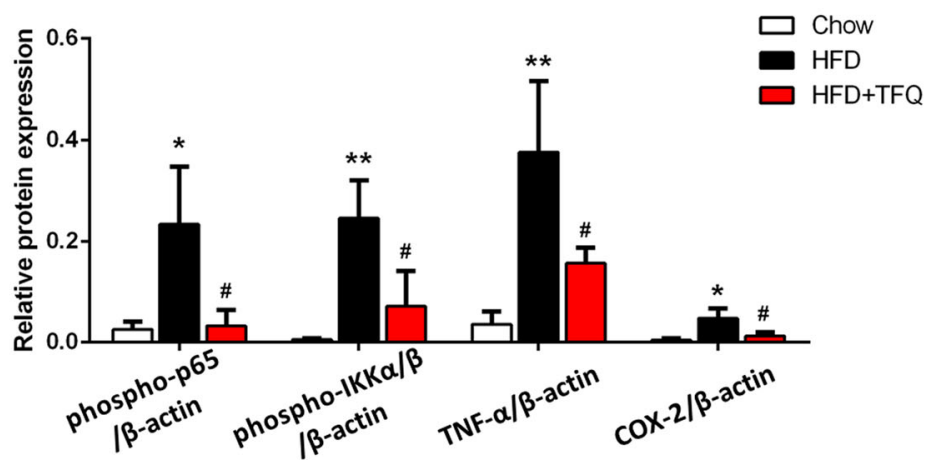

Fig. 2 The effect of TFQ on intestinal inflammation in HFD-fed mice. a Serum LPS level in mice. Data were expressed as the mean \pm SD $(n=12)$. b The mRNA expression of Cldn3 and Ocln were assessed by RT-PCR. c The protein levels of phospho-p65, phospho-IKKa/ $\beta$, TNF- $a$, and COX-2 were assessed by western blot analysis. $\mathbf{d}$ The density of bands was normalized to $\beta$-actin. Values were expressed as mean $\pm \operatorname{SD}(n=3)$. ${ }^{*} p<0.05$, ${ }^{* *} p<$ 0.01 , vs. chow group; ${ }^{\#} p<0.05,{ }^{\# \#} p<0.01$, vs. HFD group

flavonoids are a sort of the main components of Quzhou Fructus Aurantii extract, we investigated the effect of TFQ on HFD-induced obesity mouse model. The results showed that the administration of TFQ reduced body weight increasing and body lipid accumulation. TFQ gavage ameliorated the progression of fatty liver, as revealed by decreased hepatic triglyceride accumulation. TFQ improved insulin tolerance (lowered HOMA-IR index) and enhanced glucose utilization (decreased glucose AUC in OGTT), which may explain its preventive effects on visceral obesity and liver steatosis in part.
Under the influence of a high-fat diet, the changing of intestinal microbiota activates immune cells residing in the gut to release of inflammatory cytokine, which further impairs tight junction proteins and intestinal barrier function ${ }^{29}$. Consequently, LPS penetrates into the circulatory system and causes metabolic endotoxaemia, triggering obesity associated systemic inflammation and other metabolic disorders ${ }^{28}$. Consistent with these reports, our results indicated that HFD feeding activated NF- $\mathrm{KB}$ signaling pathway and elevated the expression of inflammatory cytokines (e.g., TNF $\alpha$ and COX-2), 

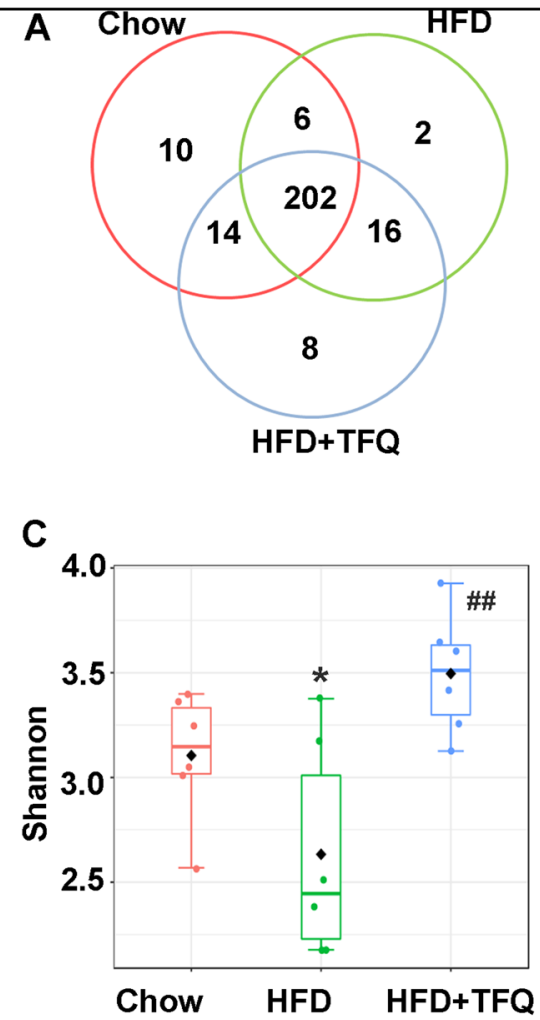

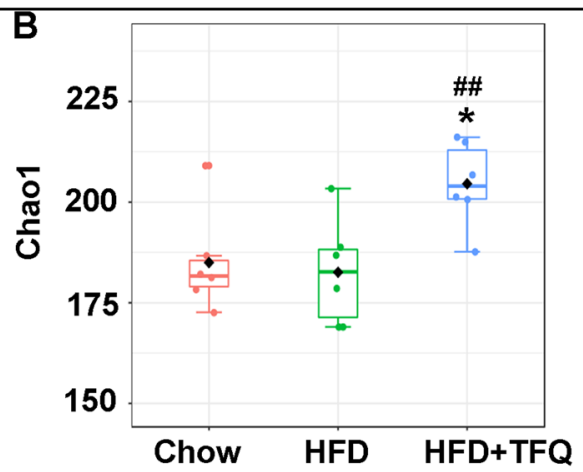

D

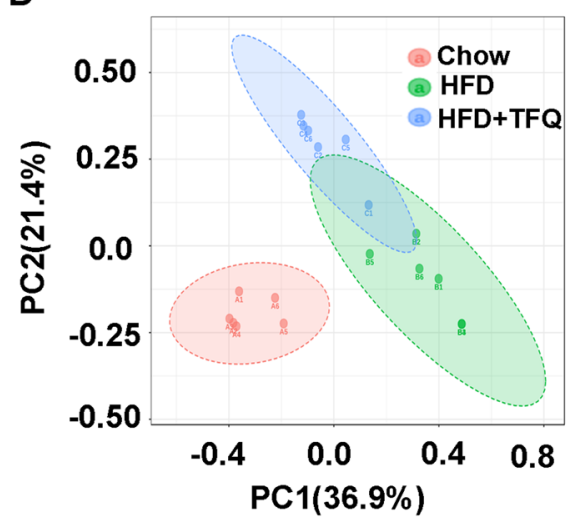

Fig. 3 TFQ modulates the overall composition of bacterial community. a A Venn diagram showing shared and unique OTUs among the three groups. The Chao 1 index (b) and the Shannon index (c) for alpha diversity estimation. d Principal-coordinate analysis (PCoA) plot showing the similarity relations among the three groups. $n=6$ per group. ${ }^{*} p<0.05,{ }^{* *} p<0.01$, vs. chow group; ${ }^{\#} p<0.05,{ }^{\# \#} p<0.01$, vs. HFD group

followed by impaired tight junction proteins and increased serum LPS penetration. TFQ treatment dramatically reduced the serum concentration of LPS induced by HFD feeding. The underlying mechanism might be multiplex. On one hand, flavonoids are reported to effectively suppress the TLR4/NF- $\mathrm{kB}$ signaling pathway and the subsequent pro-inflammatory cytokine expression ${ }^{30}$. On the other hand, flavonoids regulate the expression and assembly of tight junction proteins by influencing tyrosine kinases and $\mathrm{PKC} \delta^{31}$. According to our observation, the flavonoid-rich TFQ could exert its effect on intestinal inflammation and intestinal barrier integrity in HFD-induced obesity mouse model via both of the regulatory pathways mentioned above.

Several lines of evidence suggest that gut microbiota play an important role in the development of obesityassociated pathologies ${ }^{5,6}$. Greater diversity of the intestinal microbiota appears to be negatively correlated with abnormal weight gain and type 2 diabetes ${ }^{8,9}$. In this study, TFQ supplementation significantly elevated alpha diversity and reshaped the structure of gut microbiota. Previous survey showed obese people had about 20\% more Firmicutes and 90\% less Bacteroidetes than lean people $^{32}$. Firmicutes metabolized sugars more efficiently than Bacteroidetes, which was in favor of energy resorption and obesity. The higher ratio of Firmicutes to Bacteroidetes thus means the probability to more calorie intake and overweight ${ }^{33,34}$. TFQ treatment prominently decreased the ratio of Firmicutes to Bacteroidetes, which might be another mechanism to explain the improvement of TFQ in HFD-induced obesity and insulin resistance.

At the genus level, the HFD significantly reduced the number of Akkermansia and Alistipes. However, TFQ supplement recovered these beneficial bacteria. Akkermansia is a mucin-degrading bacterium of the phylum Verrucomicrobia commonly found in human gut ${ }^{35}$. Nowadays, it is accepted that Akkermansia is a kind of probiotics and the reduction of gut Akkermansia is associated with obesity related metabolic syndrome, albeit the exact mechanisms have not been fully elucidated. According to the literatures, Akkermansia using mucins as energy source stimulates goblet cells to produce mucus, which enhances mucus layer thickness and intestinal barrier ${ }^{36}$. From this point of view, TFQ 

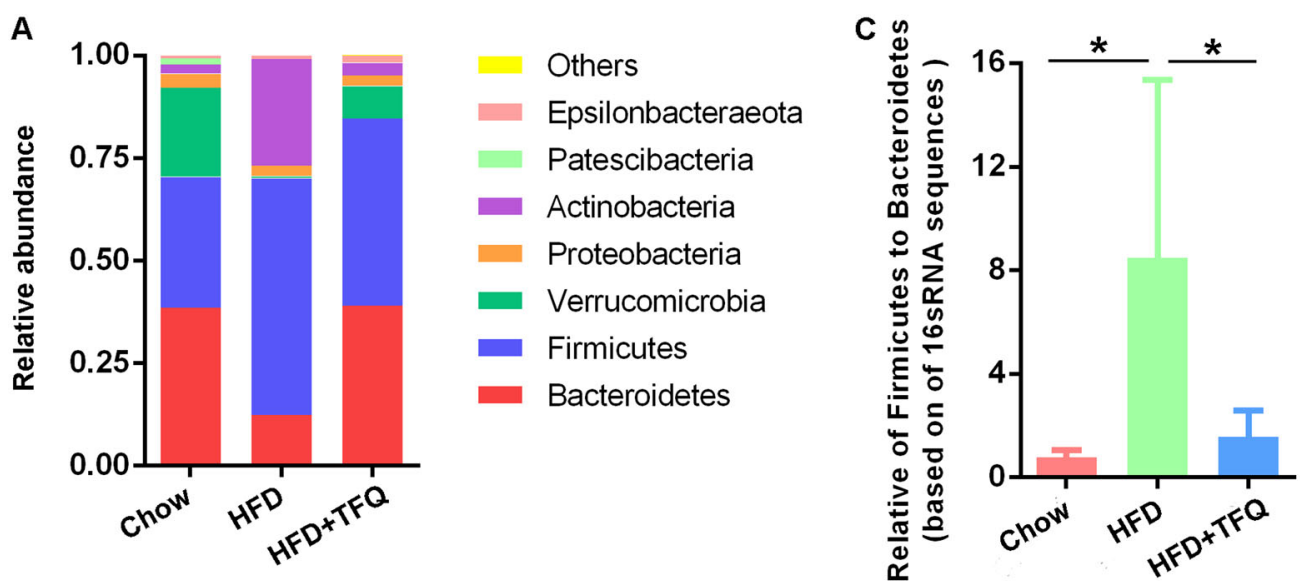

B

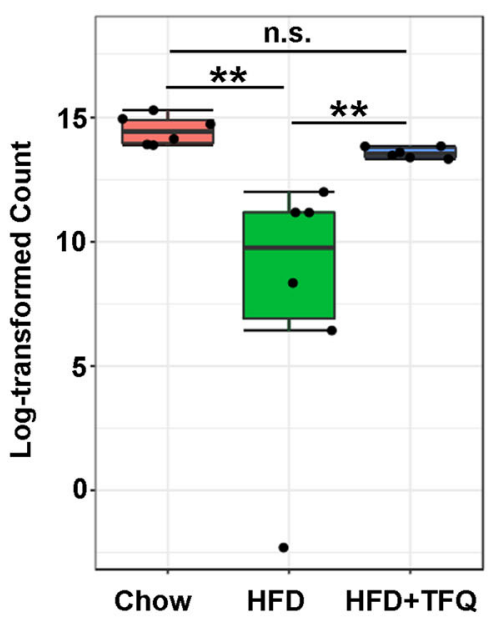

Bacteroidetes

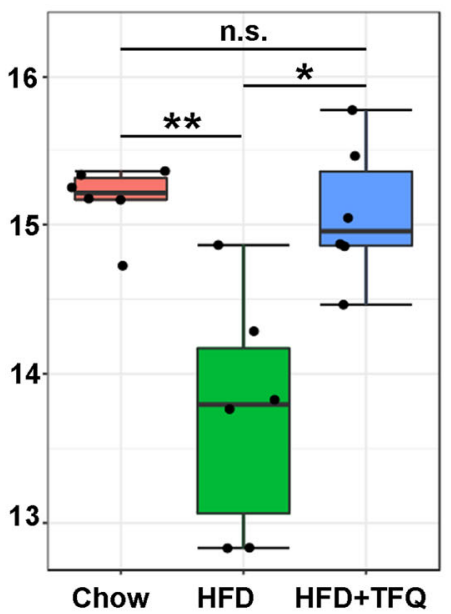

Actinobacteria

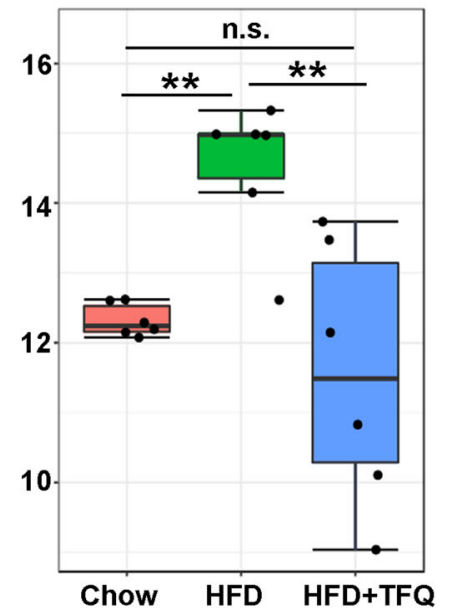

Fig. 4 The change of the gut microbiota structure at the phylum level. a Histogram of the relative abundance of the dominant bacterial phyla. $\mathbf{b}$ TFQ reversed the specific bacterial phyla. c The ratio of Firmicutes to Bacteroidetes. Values were expressed as mean \pm SD. $n=6$ per group. ${ }^{*} p<0.05$; ${ }^{* *} p<0.01$; n.S., no significance

stimulated increase in Akkermansia population might contribute to the reduction of gut permeability and LPS leakage. Studies have shown that many flavonoid-rich substances such as cranberry extract and green tea leaves are also able to increase the proportion of Akkermansia in animal or human gut ${ }^{27,37}$. Alistipes is a Bacteroidetes member of the family Rickenellaceae. Studies have demonstrated a negative correlation between obesity and the abundance of Alistipes in the gut $^{38}$. The increase of Alistipes in the gut of TFQ treated HFD-fed mice may aid in its obesity prevention effect. In addition, HFD increased the abundance of Dubosiella, Faecalibaculum and Lactobacillus, while TFQ treatment normalized these bacteria close to the level of chow-fed group. Although the effects of these bacteria on lipid and glucose metabolism are not welldocumented, those might be conducive to keep the energy homeostasis of the body as well. Therefore, we conclude TFQ is beneficial to the balance of glycolipid metabolism and prevention of obesity probably through restoration of specific flora to a normal healthy baseline.

In summary, TFQ prevents HFD-induced obesity, insulin resistance and liver steatosis. These effects are related to the upregulation of tight junction proteins and the reduction of metabolic endotoxemia. Our study further suggests that TFQ could modulate gut microbiota by reducing the ratio of Firmicutes to Bacteroidetes, and modulating the relative abundance of some genera, including Akkermansia, Alistipes, Dubosiella, Faecalibaculum, and Lactobacillus. These findings support a prebiotic role of TFQ as a dietary supplement for the intervention of gut dysbiosis and obesity-related metabolic disorders. 

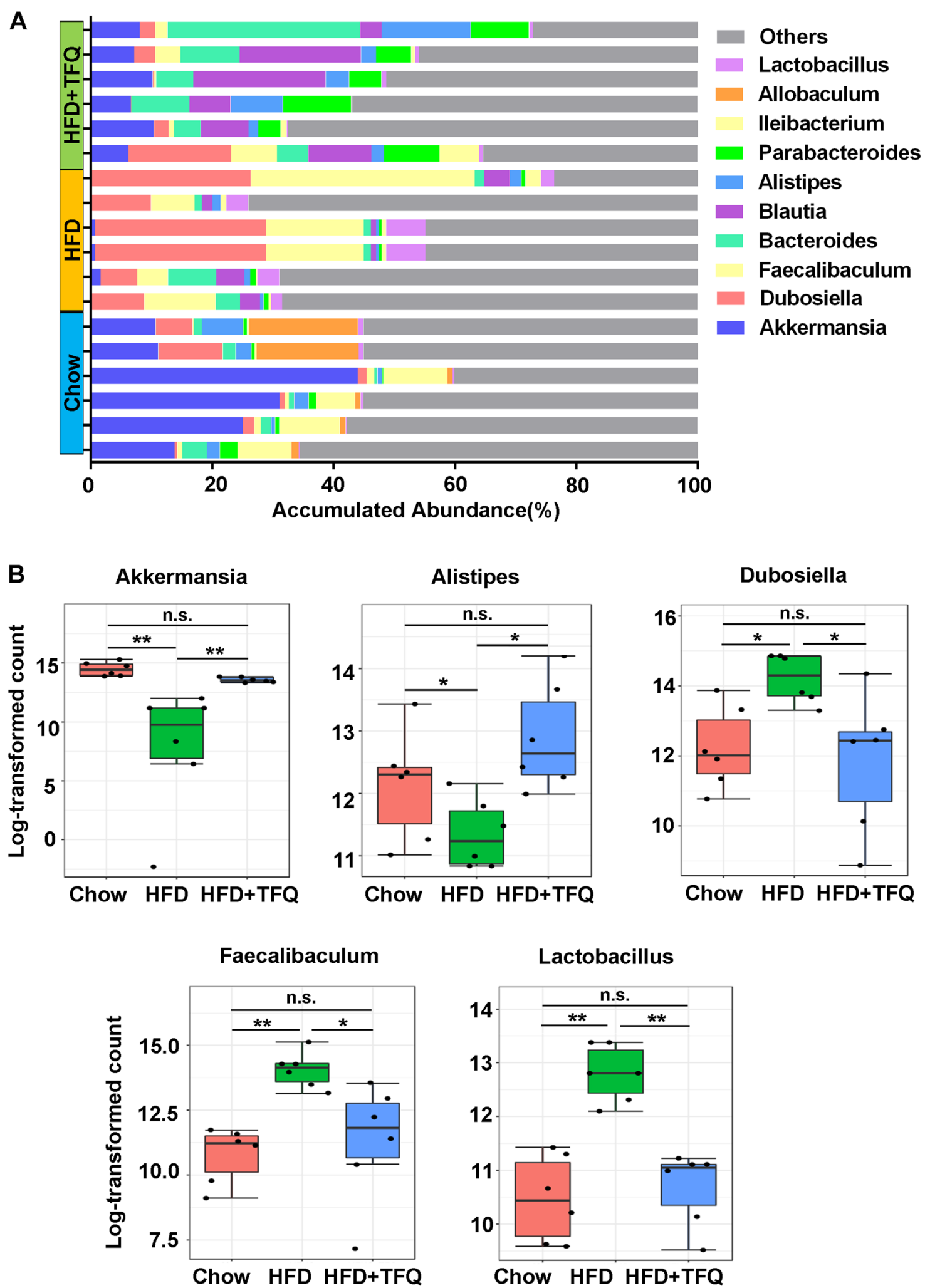

Fig. 5 The change of the gut microbiota structure at the genus level. a The accumulative abundance of the top 10 genera among the different groups. b TFQ reversed the specific bacterial genera. $n=6$ per group. ${ }^{*} p<0.05$; ${ }^{*} p<0.01$; n.s., no significance 


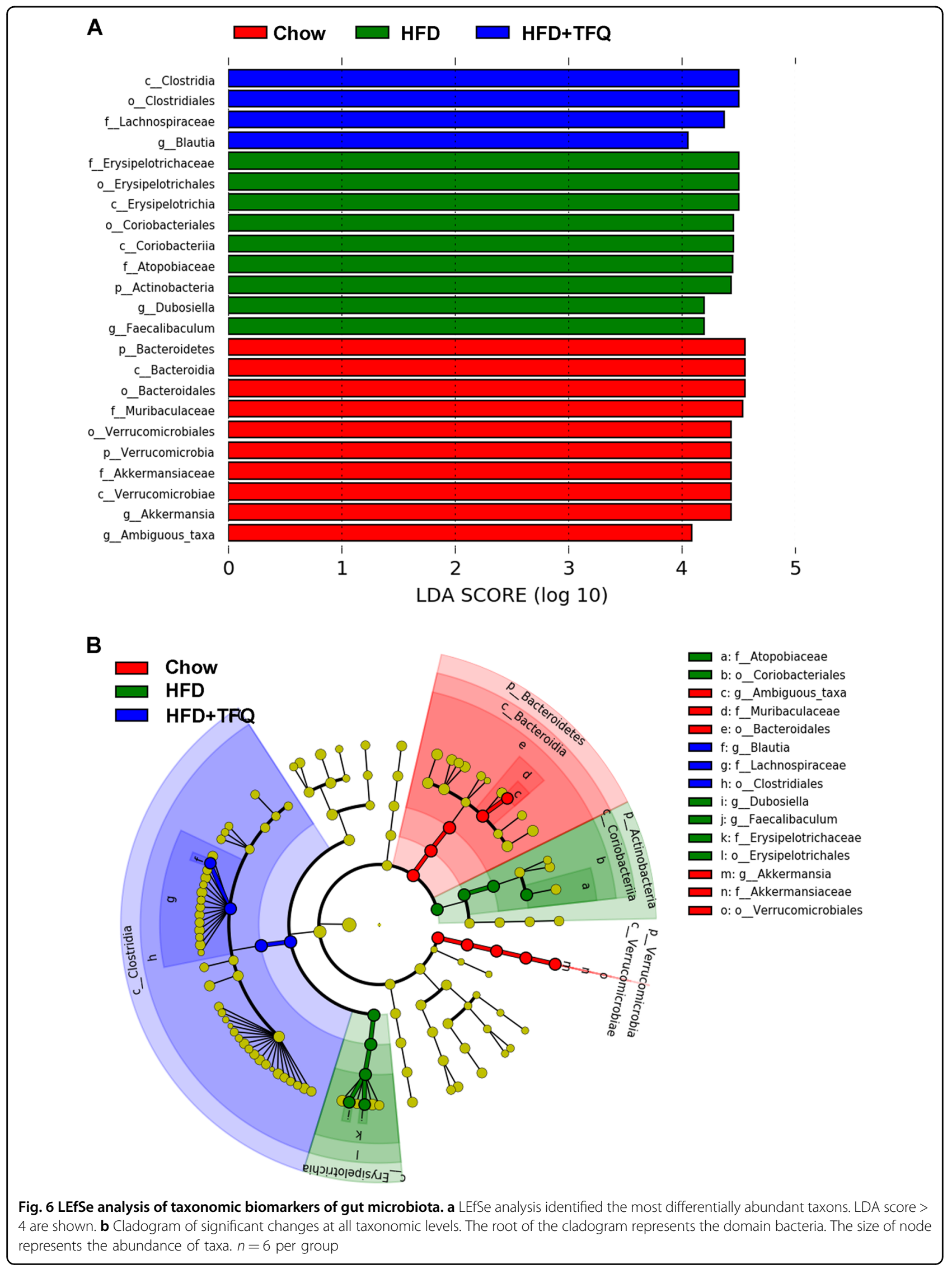




\section{Acknowledgements}

We would like to thank Dr. Jingqi Fu for advices of experimental design. This work was supported by the Suitable Technology Promotion Center New Technology and Product Research and Development Projects [2019PY089], the Science and Technology Projects of Quzhou [2018K20, 2016J016], and the Chinese Medicine Science Foundation of Zhejiang Province [2018ZB134]. All data generated or analyzed during this study are included in this published article and its supplementary information files. Supplementary information is available at NUTD's website.

\section{Author details}

'Department of Clinical Laboratory, Quzhou People's Hospital, 324000 Quzhou, China. ${ }^{2}$ Department of Core Facility, Quzhou People's Hospital, 324000 Quzhou, China. ${ }^{3}$ Department of Pharmacy, Quzhou People's Hospital, 324000 Quzhou, China. ${ }^{4}$ Department of Drug Analysis Center, Quzhou Institute for Food and Drug Control, 324000 Quzhou, China. ${ }^{5}$ Medical School, Zhejiang University, 310058 Hangzhou, China. ${ }^{6}$ Wenzhou Medical University, 325000 Wenzhou, China

\section{Author's contributions}

Participated in research design: F.Z., Y.B., S.W., L.L.; Conducted experiments: Y.B., S.W., X.W., Y.W., X.F., H.S., X.Z.; Performed experimental data analysis: Y.B., S.W. F.Z., L.L., X.Z:; Performed bioinformatic data analysis: Y.B., X.Z; Wrote or contributed to the writing of the paper: Y.B., S.W., F.Z.

\section{Conflict of interest}

The authors declare that they have no conflict of interest.

\section{Publisher's note}

Springer Nature remains neutral with regard to jurisdictional claims in published maps and institutional affiliations.

Supplementary Information accompanies this paper at (https://doi.org/ 10.1038/s41387-019-0097-6)

Received: 19 June 2019 Revised: 4 October 2019 Accepted: 4 October 2019 Published online: 23 October 2019

\section{References}

1. Hossain, P., Kawar, B. \& El Nahas, M. Obesity and diabetes in the developing world-a growing challenge. N. Engl. J. Med. 356, 213-215 (2007).

2. $\mathrm{Xu}, \mathrm{H}$. et al. Chronic inflammation in fat plays a crucial role in the development of obesity-related insulin resistance. J. Clin. Invest. 112, 1821-1830 (2003).

3. Tang, W. H. W., Li, D. Y. \& Hazen, S. L. Dietary metabolism, the gut microbiome, and heart failure. Nat. Rev. Cardiol. 16, 137-154 (2019).

4. Osborn, O. \& Olefsky, J. M. The cellular and signaling networks linking the immune system and metabolism in disease. Nat. Med. 18, 363-374 (2012).

5. Ridaura, V. K. et al. Gut microbiota from twins discordant for obesity modulate metabolism in mice. Science 341, 1241214 (2013).

6. Baothman, O. A., Zamzami, M. A., Taher, I., Abubaker, J. \& Abu-Farha, M. The role of Gut Microbiota in the development of obesity and diabetes. Lipids Health Dis. 15, 108 (2016).

7. Liang, Y. et al. Organophosphorus pesticide chlorpyrifos intake promotes obesity and insulin resistance through impacting gut and gut microbiota. Microbiome 7, 19 (2019).

8. Chassaing, B. et al. Dietary emulsifiers impact the mouse gut microbiota promoting colitis and metabolic syndrome. Nature 519, 92-96 (2015).

9. Wolf, K. J. \& Lorenz, R. G. Gut microbiota and obesity. Curr. Obes. Rep. 1, 1-8 (2012).

10. Cani, P. D. et al. Metabolic endotoxemia initiates obesity and insulin resistance. Diabetes 56, 1761-1772 (2007)

11. Alam, M. A. et al. Effect of citrus flavonoids, naringin and naringenin, on metabolic syndrome and their mechanisms of action. Adv. Nutr. 5, 404-417 (2014).

12. Liu, Y. J. et al. Dietary flavonoids intake and risk of type 2 diabetes: a metaanalysis of prospective cohort studies. Clin. Nutr. 33, 59-63 (2014).
13. Guasch-Ferre, M., Merino, J., Sun, Q., Fito, M. \& Salas-Salvado, J. Dietary polyphenols, mediterranean diet, prediabetes, and type 2 diabetes: a narrative review of the evidence. Oxid. Med. Cell Longev. 2017, 6723931 (2017).

14. van Duynhoven, J. et al. Metabolic fate of polyphenols in the human superorganism. Proc. Natl Acad. Sci. USA 108(Suppl 1), 4531-4538 (2011).

15. Estruel-Amades, S. et al. Hesperidin effects on gut microbiota and gutassociated lymphoid tissue in healthy rats. Nutrients 11, 324 (2019).

16. Porras, D. et al. Functional Interactions between gut microbiota transplantation, quercetin, and high-fat diet determine non-alcoholic fatty liver disease development in germ-free mice. Mol. Nutr. Food Res. 63, e1800930 (2019).

17. Cheng, L. et al. A metagenomic analysis of the modulatory effect of Cyclocarya paliurus flavonoids on the intestinal microbiome in a high-fat dietinduced obesity mouse model. J. Sci. Food Agric. 99, 3967-3975 (2019).

18. Alkhalidy, H., Wang, Y. \& Liu, D. Dietary flavonoids in the prevention of T2D: an overview. Nutrients 10, 438 (2018).

19. Li, L. et al. Role of Quzhou Fructus Aurantii extract in preventing and treating acute lung injury and inflammation. Sci. Rep. 8, 1698 (2018).

20. Wang, X. et al. Study on the hypoglycemic activity of different components of quzhiqiao in vitro and analysis of four flavonoids. Chin. J. Mod. Appl Pharm. 34, 1418-1423 (2017)

21. Brunt, E. M., Janney, C. G., Di Bisceglie, A. M., Neuschwander-Tetri, B. A. \& Bacon, B. R. Nonalcoholic steatohepatitis: a proposal for grading and staging the histological lesions. Am. J. Gastroenterol. 94, 2467-2474 (1999).

22. Wang, S. W. et al. Astilbin ameliorates cisplatin-induced nephrotoxicity through reducing oxidative stress and inflammation. Food Chem. Toxicol. 114 227-236 (2018).

23. Wang, S. W. et al. Cinobufacini ameliorates dextran sulfate sodium-induced colitis in mice through inhibiting M1 macrophage polarization. J. Pharm. Exp. Ther. 368, 391-400 (2019).

24. Li, Y. et al. Comparative analysis of the gut microbiota composition between captive and wild forest musk deer. Front. Microbiol. 8, 1705 (2017).

25. Dhariwal, A. et al. MicrobiomeAnalyst: a web-based tool for comprehensive statistical, visual and meta-analysis of microbiome data. Nucleic Acids Res. 45 W180-W188 (2017)

26. Du, J. et al. Betaine supplementation enhances lipid metabolism and improves insulin resistance in mice fed a high-fat diet. Nutrients 10, 131 (2018).

27. Anhe, F. F. et al. A polyphenol-rich cranberry extract protects from dietinduced obesity, insulin resistance and intestinal inflammation in association with increased Akkermansia spp. population in the gut microbiota of mice. Gut 64, 872-883 (2015).

28. Cani, P. D. et al. Changes in gut microbiota control metabolic endotoxemiainduced inflammation in high-fat diet-induced obesity and diabetes in mice. Diabetes 57, 1470-1481 (2008)

29. Gil-Cardoso, K. et al. Effects of flavonoids on intestinal inflammation, barrier integrity and changes in gut microbiota during diet-induced obesity. Nutr. Res. Rev. 29, 234-248 (2016)

30. Dou, W. et al. Protective effect of naringenin against experimental colitis via suppression of Toll-like receptor 4/NF-kappaB signalling. Br. J. Nutr. 110, 599-608 (2013).

31. Suzuki, T. \& Hara, H. Role of flavonoids in intestinal tight junction regulation. J. Nutr. Biochem 22, 401-408 (2011).

32. Santacruz, A. et al. Gut microbiota composition is associated with body weight, weight gain and biochemical parameters in pregnant women. Br. J. Nutr. 104, 83-92 (2010).

33. Turnbaugh, P. J. et al. A core gut microbiome in obese and lean twins. Nature 457, 480-484 (2009).

34. De Filippo, C. et al. Impact of diet in shaping gut microbiota revealed by a comparative study in children from Europe and rural Africa. Proc. Natl Acad. Sci. USA 107, 14691-14696 (2010).

35. Derrien, M., Vaughan, E. E., Plugge, C. M. \& de Vos, W. M. Akkermansia muciniphila gen. nov., sp. nov., a human intestinal mucin-degrading bacterium. Int. J. Syst. Evol. Microbiol. 54, 1469-1476 (2004).

36. Everard, A. et al. Cross-talk between Akkermansia muciniphila and intestinal epithelium controls diet-induced obesity. Proc. Natl Acad. Sci. USA 110 9066-9071 (2013).

37. Axling, U. et al. Green tea powder and Lactobacillus plantarum affect gut microbiota, lipid metabolism and inflammation in high-fat fed C57BL/6J mice Nutr. Metab. 9, 105 (2012).

38. Verdam, F. J. et al. Human intestinal microbiota composition is associated with local and systemic inflammation inobesity. Obesity 21, E607-E615 (2013). 